\title{
The subjective tempo difference between interaural and monaural sequences as a function of sequence length
}

\author{
GERT ten HOOPEN and SIMONE AKERBOOM \\ University of Leiden, Leiden, The Netherlands
}

\begin{abstract}
Previous research has demonstrated that the subjective tempo of sequences of clicks that alternate between ears is slower than that of nonalternating sequences. Although the stimulus onset asynchronies (SOAs) between the clicks are the same in both conditions, their perceptual onset asynchronies (POAs) differ by $25 \mathrm{msec}$ at all SOA values between 40 and $2,130 \mathrm{msec}$. It has been suggested that this subjective tempo difference originates only after a few clicks have been processed. The present study shows this not to be the case: The POA difference between interaural and monaural click sequences could also be established with sequences comprising only a few clicks.
\end{abstract}

If a sequence of auditory signals is presented interaurally (i.e., each consecutive signal arrives at the other ear), such a sequence has a slower apparent tempo than a monaural sequence (Axelrod \& Guzy, 1968; Axelrod, Guzy, \& Diamond, 1968). We devoted a series of studies to this phenomenon and were finally able to establish that the amount of the subjective tempo difference between interaural and monaural sequences was $24 \mathrm{msec}$ (ten Hoopen, Vos, \& Dispa, 1982). Although the value of $24 \mathrm{msec}$ can be arrived at by three different methods, we will discuss only the most powerful one.

Listeners were required to respond as fast as possible to the unpredictable end of a tone sequence. The so-called stop-reaction time (stop-RT) comprises two durations. The first duration is the perceptual onset asynchrony (POA) between the tones. This is the criterion time that the subject sets for himself after each tone, and, if no further tone arrives during that time, he initiates his response, a process taking an additional amount of time-the second duration. If it is assumed that response initiation time is invariant with the presentation mode (interaural vs. monaural), then one can interpret differences between stop RTs as differences between POAs.

In the ten Hoopen et al. (1982) study, the difference between interaural and monaural POA turned out to be $24 \mathrm{msec}$. In addition, this difference was invariant with the stimulus onset asynchrony (SOA)

This study was made possible by a grant from the Netherlands Organization for the Advancement of Pure Research (ZWO). Thanks are extended to Hanneke van Mier, Machiel Hermans, Felix Julien, and Rob van Dam for assisting in the experiments. Requests for reprints may be sent to Gert ten Hoopen, Unit of Experimental Psychology, Hooigracht 15, 2312 KM Leiden, The Netherlands. between the tones, at least in the range of $125-250 \mathrm{msec}$ SOA. The results of a further study at our laboratory (Akerboom, ten Hoopen, Olierook, \& van der Schaaf, 1983) showed that the invariance of the POAdifference holds even for an SOA range of 40$2,130 \mathrm{msec}$. The POA difference established in this latter study was $25 \mathrm{msec}$-almost the same as that found in the previous study. In other words, no matter how fast or slow the objective tempo of the sequence is, the subjective difference between interaural and monaural tempi remains $25 \mathrm{msec}$. Figures 1 and 2 represent our conception of the difference between interaural and monaural POA. The essence of this representation is that interaural events are stretched out in auditory memory for $25 \mathrm{msec}$ farther than monaural events, although for both conditions the SOA is the same.

In 1972, Guzy and Axelrod published an experiment in which their subjects had to count the number of clicks in sequences of varying length. In addition, the stimulus onset asynchrony (SOA) between the clicks and the presentation mode were varied. Clicks were presented monaurally (all clicks to the same ear) or interaurally. During interaural presentation, the number of clicks was undercounted relative to the monaural presentation. The authors explain this performance deficit by time-consuming and fatigable attention-switching between auditory locations. Ten Hoopen and Vos (1980) demonstrated that Guzy and Axelrod based this explanation on a wrong analysis of their data. I attempted to point out that the undercounting of interaural clicks could not be accounted for by an attention-switching mechanism (ten Hoopen, 1982). The explanation of this undercounting can be given better in terms of the POA differences between interaural and monaural sequences (ten Hoopen, Vos, \& Dispa, 1982). 


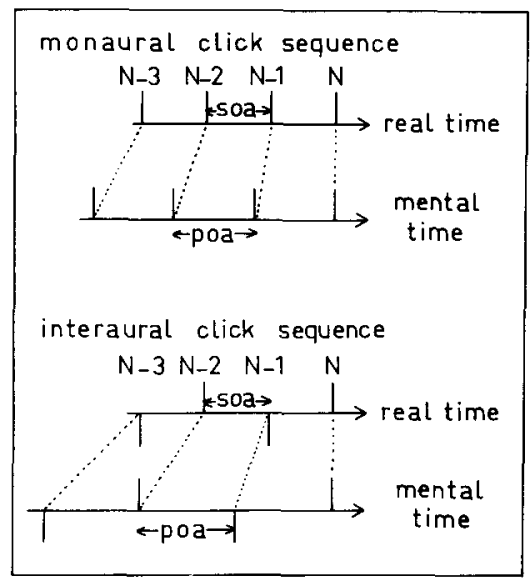

Figure 1. Monaural and interaural sequences with the same stimulus onset asynchrony (SOA) between tones (in real time) have different perceptual onset asynchronies (POA) in mental time. When listening "back" in echoic memory, interaural tones appear more widely spaced.

Nevertheless, Guzy and Axelrod (1972) reported two informal, but important, observations, which formed the genesis of their article but which have not yet been systematically investigated. Their observations were the following: (a) rapid interaural click sequences appear to slow down only after a few clicks have been presented, and (b) the slowing down of the interaural sequences appears earlier in faster sequences than in slower ones. The observation by Guzy and Axelrod that it takes some time before the tempo of a location-alternating sequence slows down brings to mind a recently reported effect of frequency-alternating tone sequences.

In the last decade, an increasing number of studies has investigated the perceptual effects of alternating tone sequences between different frequencies (van Noorden, 1975; see also Bregman, 1978a, and McAdams \& Bregman, 1979, for reviews). If a tone sequence is alternated between frequencies, one of two possible phenomena will occur. Either the sequence is heard as a coherent whole or it is heard as itwo separate streams of tones, one of high tones and the ether of low tones. Which sensation arises depends on the SOA and frequency interval between the tones and, for some combinations, also on what the listener wants to hear. Van Noorden (1975) has systematically investigated the combinations of SOA and frequency interval that cause the percepts of coherence and streaming.

There is some evidence that listeners need a few alternating tones before streaming is perceived. Bregman (1978b) required his listeners to speed up the rate of a frequency-alternating tone sequence until streaming occurred. The tone sequence could be continuous or could contain silent 4-sec gaps. The number of tones between the gaps could be 4,8 , or
16. It was found that the more often the silences occurred, the faster the tones had to be alternated before streaming was perceived. This result, according to Bregman (1978a), can be interpreted as follows: the listener begins by constructing a single stream percept but splits the sequence when the evidence becomes compelling - "In short, it takes some time for a two stream interpretation to emerge"' (p. 67).

In the same vein, a possible slowing down of the rate of interaural sequences towards a stable value, as suggested by Guzy and Axelrod (1972), might be the result of a tempo processor that starts gathering evidence when the location-alternating sequence begins. By "tempo processor," we mean the auditory mechanism that transforms the real (objective) times between the sound stimuli, that is, the SOAs, into subjective times between the sound percepts, that is, the POAs.

We translated the informal observations ( $a$ and $b$ ) made by Guzy and Axelrod in terms of our POA concept: (a) the POA difference between interaural and monaural click sequences should be nil when the sequences comprise only a few clicks, but should increase to a 25-msec POA difference with increasing number of clicks, and (b) this buildup of the POA difference to $25 \mathrm{msec}$ should occur earlier with faster sequences.

\section{EXPERIMENT 1}

\section{Method}

Subjects. Forty-eight female and male students from the University of Leiden, aged 17-25 years, participated in the experiment and were paid for their services. Left- and right-ear thresholds were screened, the rejection criterion being a loss of more than $15 \mathrm{~dB}$ or a difference between left and right of more than $10 \mathrm{~dB}$. Only subjects passing this criterion participated.

Stimuli and Design. Interaural and monaural click sequences were generated by means of Coulbourn Instruments modules under the control of a minicomputer. The clicks were pure sinewave tones of $750 \mathrm{~Hz}$ with a duration of $10 \mathrm{msec}$ and rise and fall times of 2 msec each. The SOA between the clicks was varied be-

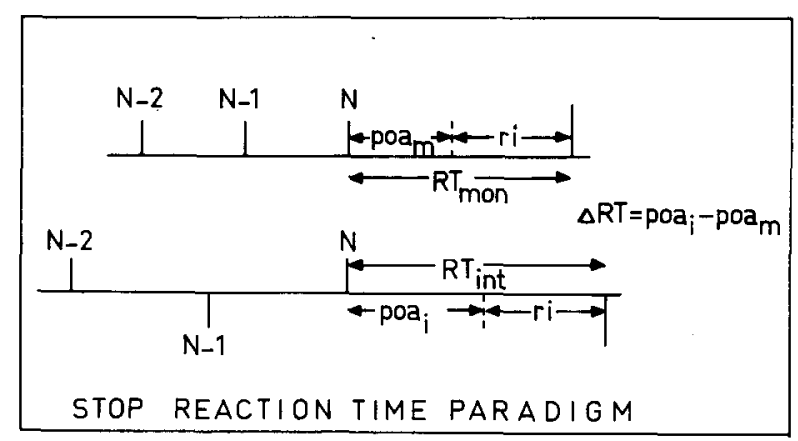

Figure 2. Illustration of the stop-RT paradigm, which shows that the difference in time between detection of the end of interaural and monaural tone sequences $\left(\mathbf{R T}_{\text {int }}-\mathbf{R T}_{\text {mon }}\right.$ ) represents the difference between interaural and monaural perceptual onset asynchronies (POA, - POA $)$. 
tween $50,100,150,200,300,400,500$, and $600 \mathrm{msec}$. The number of clicks in a sequence could be $2,3,4,5,6,7,8,9,10,11,12$, or 13 .

Thus, there were 2 (interaural vs. monaural) $\times 8$ (SOAs) $\times$ 12 (number of clicks) $=192$ different click sequences that were blocked with respect to presentation mode and SOA. In each of the 16 blocks, the eight sequences were randomized. The sequences were separated by a silent response interval of $2.5 \mathrm{sec}$.

Procedure and Apparatus. The subjects were tested individually in a sound-attenuated booth (Siemens) after they passed the screening (pure-tone audiometer, Peekel D 66). They received ample training before being presented with the 16 experimental blocks. These blocks were incompletely counterbalanced according to a Latin square, and to each permutation three subjects were ascribed.

The subjects were instructed to respond by depressing a pushbutton under the dominant index finger as soon as they were sure that a click sequence was over. The stop-RT, that is, the elapsed time between the end of the last click and the buttonpress, was registered by the computer. All apparatus, except for the headphones (AKG-Cardan K140) and the pushbutton, were located outside the booth.

\section{Results and Discussion}

From the introduction, it will be recalled that the first informal observation (a) of Guzy and Axelrod was that interaural click sequences appeared to slow down only after a few clicks had been presented. In terms of our stop-RT paradigm, this implies that the difference between interaural and monaural stop-RT should increase from 0 to about $25 \mathrm{msec}$ with an increasing number of clicks in the sequence. Figure 3 clearly shows this not to be the case. On the contrary, the difference at the shortest sequences is even larger. This interaction effect between presentation mode and number of clicks was significant $[F(11,517)=$ $7.39, \mathrm{p}<.0001]$, and we shall return to this result further on. Thus, it appears that observation a is not supported by our data. It might be, however, that we obscured the picture by averaging over all SOAs. Therefore, in Figure 4, we split Figure 3 up into four separate pictures, with one averaged over the SOAs of 50 and $100 \mathrm{msec}$, one over the SOAs of 150 and $200 \mathrm{msec}$, one over the SOAs of 300 and $400 \mathrm{msec}$, and one over the SOAs of 500 and $600 \mathrm{msec}$. For

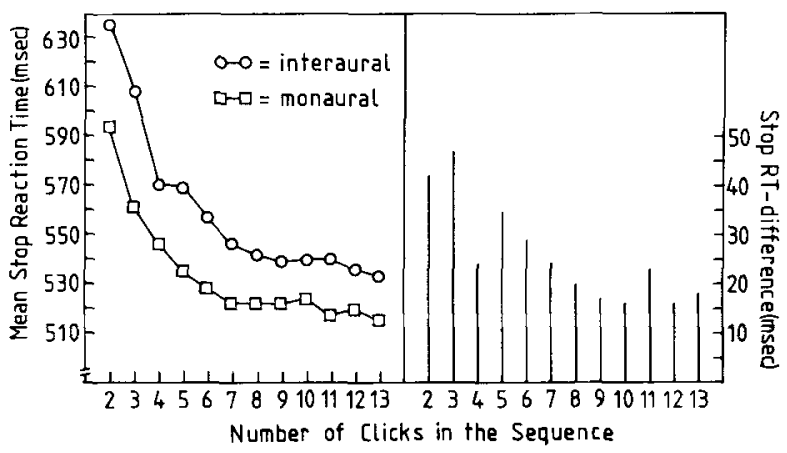

Figure 3. Mean stop-RTs as a function of number of clicks and presentation mode (left panel). Differences between interaural and monaural stop-RTs dependent on number of clicks (right panel).

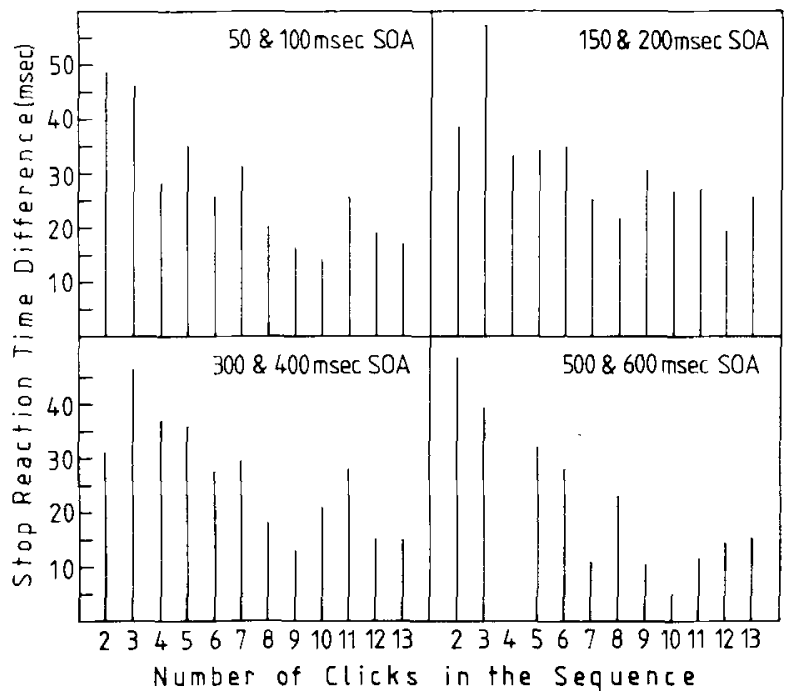

Figure 4. Differences between interaural and monaural stopRTs dependent on number of clicks and for four ranges of SOA.

clarity of illustration, only the stop-RT differences are plotted in these figures. As one can readily see, the overall pattern of Figure 3 is not changed very much when taking the value of the SOA into account. (The fact that there is no stop-RT difference at the level of 4 clicks in the right lower panel of Figure 4 is probably due to chance.) Thus, even at very short SOAs and with only a few clicks ( 2 or 3 ), there is a pronounced difference between interaural and monaural stop-RT. Hence, we have to reject informal observation a. Figure 4 also shows that observation b (that the slowing down of the interaural sequences occurs earlier with faster sequences) is not supported either.

Let us now return to Figure 3. As one can see, with increasing number of clicks in the sequence the stop-RTs strongly decrease up to about seven clicks and level off with longer sequences. [The main effect of number of clicks was significant: $F(11,517)=$ $174.45, \mathrm{p}<.0001]$. This certainly indicates an expectancy effect: listeners do not expect that the sequence will stop after only a few clicks. For the present purposes, this main effect is, however, not relevant. More important is the significant interaction between presentation mode and number of clicks on the stop-RT: The stop-RT differences are larger for sequences with 2-6 clicks than they are with longer sequences. The average value of the stop-RT differences at these longer sequences (7-13 clicks) amounts to $19 \mathrm{msec}$ and is a reliable estimate of the POA difference (see Figure 5). This value nicely approximates the values found in our previous studies (24 and $25 \mathrm{msec}$ ).

The fact that for short click sequences the interaural stop-RT is significantly larger than the sum of the monaural stop-RT and the "normal" POA dif- 


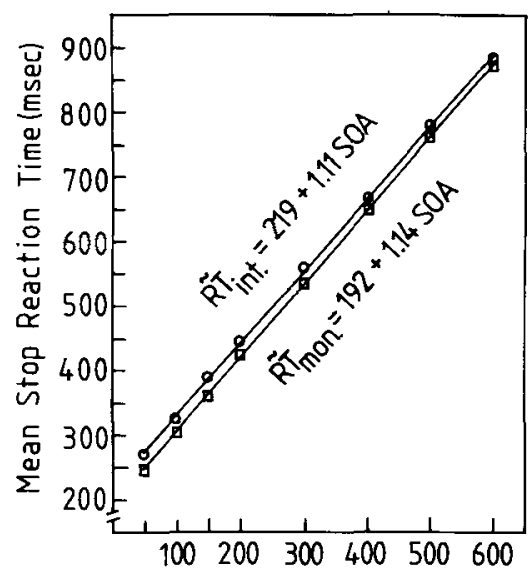

Stimulus Onset Asynchrony insed

Figure 5. Mean stop-RTs dependent on SOA and presentation mode (interaural vs, mongural) averaged over the number of click levels 7-13. The best-fitting linear regression lines are inserted (both $r^{2}$ s exceed 0.999).

ference might be due, in part, to auditory Gestalt factors. If a sequence of clicks alternates between the left and right ears, listeners possibly prefer a "whole" structure, that is, an even number of clicks. If this supposition is true, then, when the sequence contains an odd number of clicks, listeners should display a tendency to inhibit their stop-RT somewhat. Inspection of Figure 3, right panel, reveals that the differences between interaural and monaural stop-RTs are, indeed, larger for sequences containing 3 and 5 clicks than for sequences containing 2,4 , and 6. This odd-even effect disappears when the sequences comprise more than 6 clicks. Apparently, listeners then have lost track of which ear was stimulated first and consequently no longer show a preference for an even number of clicks in the sequence.

It is evident, however, that the differences between interaural and monaural stop-RTs for short evennumbered click sequences are still larger than those for relatively long sequences. That is, in addition to the main expectancy effect which enlarges stop-RTs for short sequences in the interaural as well as in the monaural condition, there is some unknown factor which enlarges stop-RTs for short interaural sequences to a greater extent.

In order to answer the question of whether this extra enlargement is caused by the range of numbers of clicks utilized, we decided to carry out a second experiment with a more restricted range of clicks, namely 2-6 clicks.

\section{EXPERIMENT 2}

\section{Method}

The method was the same as that of Experiment 1, except for the following: Twenty subjects were used, and all of them received the conditions twice. The range of numbers of clicks in the sequence was 2-6.

\section{Results and Discussion}

The mean stop-RTs dependent on presentation mode and number of clicks are plotted in Figure 6.

Like in the first experiment of the present study, there is a strong decrease in stop-RT with increasing number of clicks in the sequence. This effect of number of clicks was significant $[F(4,76)=86.93, p<.001]$, as was the interaction between presentation mode and number of clicks $[F(4,76)=5.06, p<.01]$. This interaction effect can be largely accounted for by the larger stop-RT difference between interaural and monaural conditions at the shortest click sequence. As Figure 6 (right panel) shows, the stop-RT difference at 2 clicks amounts to $40 \mathrm{msec}$, which significantly decreases to $25 \mathrm{msec}$ at a sequence with 3 clicks.

The average stop-RT difference in the range of 3-6 clicks amounts to $21.5 \mathrm{msec}$, which approximates the estimate of the POA difference from previous studies rather well.

If we compare the stop-RT differences of Experiment 2 (Figure 6, right panel) with those of Experiment 1 (Figure 3, right panel), we see a decrease of this difference at each sequence length (2-6 clicks). The decrements of the stop-RT differences amounted to $2,22,6,7$, and $12 \mathrm{msec}$ at the levels of 2-6 clicks, respectively. The decrement at the sequence of 3 clicks was significant according to a Mann-Whitney $\mathrm{U}$ test, large-sample approximation $(\mathrm{z}=3.027, \mathrm{p}<$ .0013). The lack of significance of the decrements at the levels of 4,5 , and 6 clicks is quite obvious, because in Experiment 1 the stop-RT differences at these levels already approached the "normal" POA difference $(24,34,28 \mathrm{msec}$, respectively). Hence, the reduction of the stop-RT difference by the manipulation of expectancy is bounded by the "normal" POA difference. It is not obvious, however, that shortening the range did not affect the stop-RT difference between interaural and monaural conditions at the level of 2 clicks. At present, we cannot explain this result.

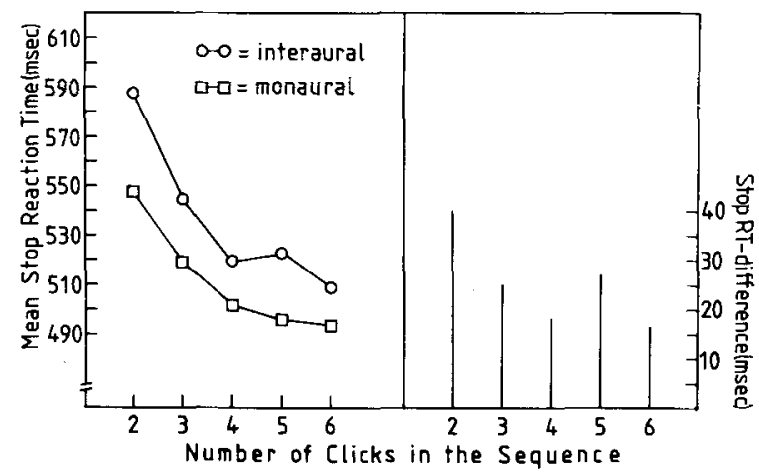

Figure 6. Mean stop-RTs as a function of number of clicks and presentation mode (left panel). Differences between interaural and monaural stop-RTs dependent on number of clicks (right panel). 
The main questions of the present study, however, have been answered unambiguously: First, the subjective tempo difference between interaural and monaural sequences originates immediately, that is, after 2 clicks have been presented. Second, the genesis of this sensation is not affected by the objective tempo of the sequences. We conclude, therefore, that the tempo processor does not need a certain amount of input of the location-alternating sequence, but that it is capable of signaling a slower rate when only two clicks are presented, regardless of whether the sequence is fast or slow.

\section{REFERENCES}

Akerboom, S. P., ten Hoopen, G., Olterook, P., \& van DER SchaAf, T. W. Auditory spatial alternation transforms auditory time. Journal of Experimental Psychology: Human Perception and Performance, 1983, 9, 882-897.

AXELrod, S., \& Guzy, L. T. Underestimation of dichotic click rates: Results using methods of absolute estimation and constant stimuli. Psychonomic Science, 1968, 12, 133-134.

Axelnod, S., Guzy, L. T., \& Diamond, I. T. Perceived rate of monotic and dichotically alternating clicks. Journal of the Acoustical Society of America, 1968, 43, 51-55.
Bregman, A. S. The formation of auditory streams. In J. Requin (Ed.), Attention and performance VII. Hillsdale, N.J: Erlbaum, 1978. (a)

Bregman, A. S. Auditory streaming is cumulative. Journal of Experimental Psychology: Human Perception and Performance, 1978, 4, 380-387. (b)

GuzY, L. T. \& AxelRod, S. Interaural attention shifting as response. Journal of Experimental Psychology, 1972, 95, 290-294.

McAdams, S., \& Bregman, A. S. Hearing musical streams. Computer Music Journal, 1979, 3, 26-43.

TEN HoOpen, G. The perceptual organization of alternating tone sequences. Unpublished doctoral dissertation, University of Leiden, The Netherlands, 1982.

TEn Hoopen, G., \& Vos, J. Attention switching is not a fatigable process: Methodological comments on Axelrod and Guzy (1972). Journal of Experimental Psychology: Human Perception and Performance, 1980, 6, 180-183.

TEn Hoopen, G., Vos, J., \& Dispa, J. Interaural and monaural clicks and clocks: Tempo difference versus attention switching. Journal of Experimental Psychology: Human Perception and Performance, 1982, 8, 422-434.

VAN Noonden, L.P.A.S. Temporal coherence in the perception of tone sequences. Unpublished doctoral dissertation, Institute of Perception Research, Eindhoven, The Netherlands, 1975.

(Manuscript received March 11, 1983; revision accepted for publication August 23, 1983.) 